\title{
Status of theoretical predictions for top pair production cross section
}

\author{
loannis Tsinikos * \\ Centre for Cosmology, Particle Physics and Phenomenology (CP3), \\ Université Catholique de Louvain, Chemin du Cyclotron 2, B-1348 Louvain-la-Neuve, Belgium, \\ Technische Universität München, James-Franck-Str. 1, D-85748 Garching, Germany \\ E-mail: ioannis.tsinikos@uclouvain.be, ioannis.tsinikos@tum.de
}

In this proceeding we review the status of theoretical predictions for top pair production and decay at differential level. Concerning stable tops we focus on the NNLO QCD+NLO EW combination, the NNLO+NNLL' calculation and the merged $t \bar{t}+t \bar{t} j$ results at NLO QCD+EW accuracy. We further present results at the decay level including off-shell effects in the semi-leptonic decay mode of the top-quark pair at NLO in QCD.

Sixth Annual Conference on Large Hadron Collider Physics (LHCP2018)

4-9 June 2018

Bologna, Italy

${ }^{*}$ Speaker. Preprint: CP3-18-57, TUM-HEP-1165/18 


\section{Introduction}

This proceeding consists of results based on a collection of recent theoretical papers discussing high precision calculations on the top-quark pair process at the LHC at the production [1-3] and the decay level [4]. The main motivation for high precision studies in the top pair production is the fact that the new $\mathrm{LHC}$ run at $13 \mathrm{TeV}$ will probe very accurately this process even at differential level. Already there are many results available for $t \bar{t}$ production beyond NLO in QCD [5-20] and including the EW corrections [3,21-35]. At the decay level the most recent works focus on the fully-leptonic decay of the top-quark pair at NLO in QCD [36], at NLO in EW [31] and the semileptonic decay at NLO in QCD [4]. In the current proceeding we will discuss the latter. We will start with stable tops at NNLO QCD + NLO EW accuracy followed by NNLO + NNLL' and we will proceed with the semi-leptonic decay of the top-quark pair.

\section{Stable tops}

The tension between theory and experiment reported for the $p_{T}(t)$ distribution at $8 \mathrm{TeV}[37,38]$ was an extra motivation for these high accuracy calculations. In [7] the NNLO QCD calculation shows the reduction of the scale uncertainty w.r.t. the NLO QCD and even at this level of accuracy the tension between experiment and theory persists with the data to show a softer spectrum than the theory at the high $p_{T}$ region.

Starting with the NNLO QCD + NLO EW calculation it is interesting to see how this distribution is affected. For the NNLO QCD part of this calculation the calculational techniques of [8] are used. The NLO EW part is included via the currently public version of the MADGRAPH5_AMC@NLO framework [39,40] already validated in [41-43]. The multiplicative ap-
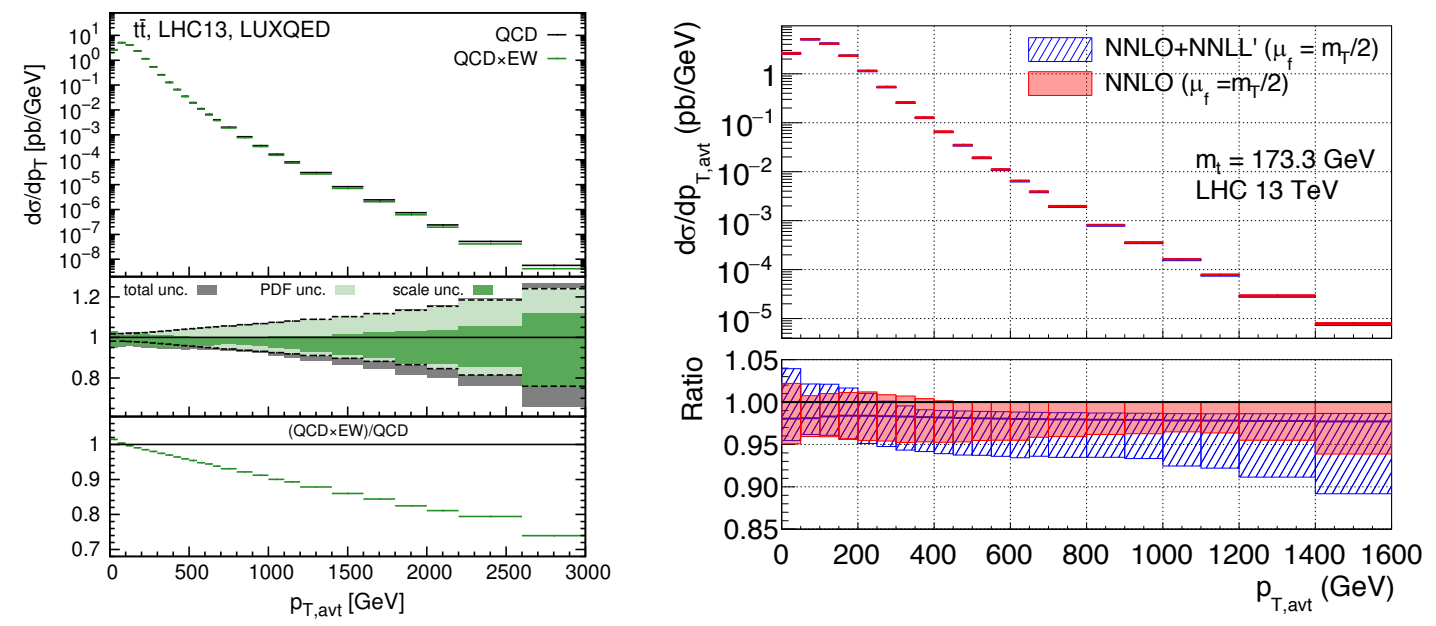

Figure 1: The QCD $\times \mathrm{EW}$ (left) and the NNLO QCD + NNLL' (right) results for the $p_{T, \text { avt }}$ distribution at $13 \mathrm{TeV}$.

proach, which is adopted here, further approximates on top of the additive one the missing higher order mixed term $O\left(\alpha_{s}^{3} \alpha\right)$ by applying the NLO QCD K-factor on the $\alpha_{s}^{2} \alpha$ perturbative order. The calculation setup and the multiplicative combination is described in detail in [1] and particularly 
the scale choices are motivated in [8]. In figure 1 we can see in the left plot the effect of the EW corrections to the NNLO QCD result at $13 \mathrm{TeV}$ for the $p_{T, \text { avt }}$ distribution. This shows that the EW corrections soften the spectrum at the high $p_{T}$ region. The study of the effects of resummation on top of the NNLO QCD results is realised in [2]. In this work the resummation of soft
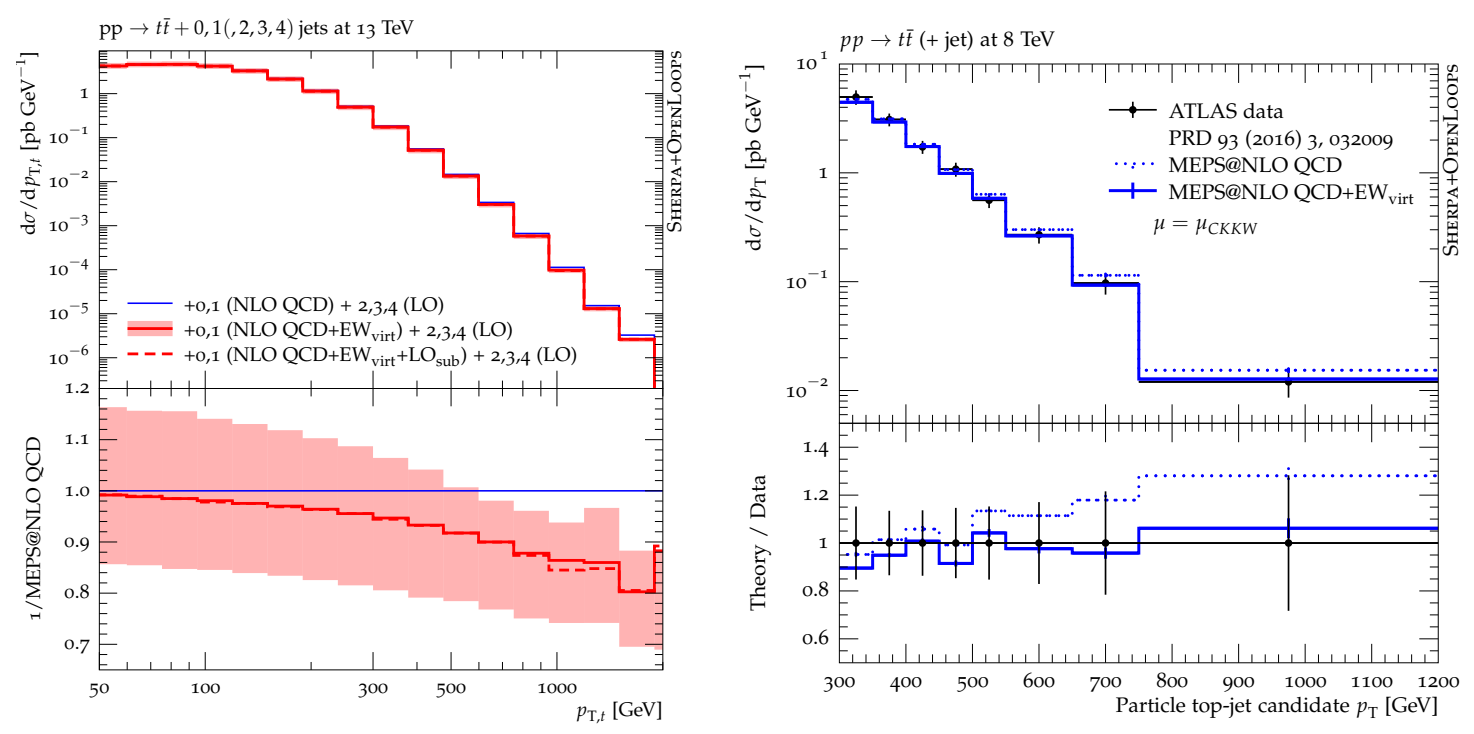

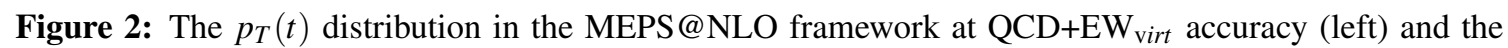
comparison with ATLAS data (right). Figure taken from [3].

and small-mass logarithms at differential level is matched to NNLO QCD in order to provide the NNLO+NNLL' prediction using the same scale choices as in [1]. Looking in figure 1 (right) we can see that for the $p_{T \text {,avt }}$ distribution the resummation also reduces the central value of the NNLO QCD. In order to go beyond the fixed order calculations another challenge is to include the shower effects keeping the top-quark pair stable. In [3] the $t \bar{t}$ and $t \bar{t} j$ samples are merged up to one jet in the MEPS@NLO framework of the SHERPA+OPENLOOPS [44-46]. In figure 2 we can see that the effect of the EW corrections softens the spectrum and brings the theory in agreement with the data from the boosted top ATLAS analysis [47]. This behaviour is in agreement with what is observed at the fixed order calculations and shown in figure 1.

\section{Off-shell effects at the decay level}

On top of the projects described in the previous section, where the tops are kept stable, there are efforts to include the top-quark decays in the calculation considering also the off-shell effects. The statistics on the new run of the LHC at $13 \mathrm{TeV}$ will provide experimentally accurate distributions on the decay products which need to be compared with the theory. Furthermore, decaying the tops introduces realistic final states where actual cuts, close to the experimental ones, can be applied. This reduces the need for the experimental analyses to unfold from particle and fiducial to parton and inclusive levels respectively, which is a Monte Carlo dependent procedure. The inclusion of the off-shell effects reveals the contribution of the non-resonant Feynman diagrams for a specific final state, which can be significant in the tails of the distributions. They are particularly 
important in observables which are used in the top-mass measurement analyses. Of course one can realise that keeping the same accuracy as in the production level is extremely challenging since the Feynman diagrams become more complicated. Recent works on the top pair production and decay are presented in $[4,31,36]$.

As stated in the introduction we will focus on the semi-leptonic decay of the top-quark pair at NLO in QCD [4]. In this work the LO $O\left(\alpha_{s}^{2} \alpha^{4}\right)$ perturbative order with the NLO QCD corrections are considered.. This order includes Feynman diagrams with at least one resonant $W$ boson and up to two top and $W$ resonant propagators. Focusing on differential distributions of the process
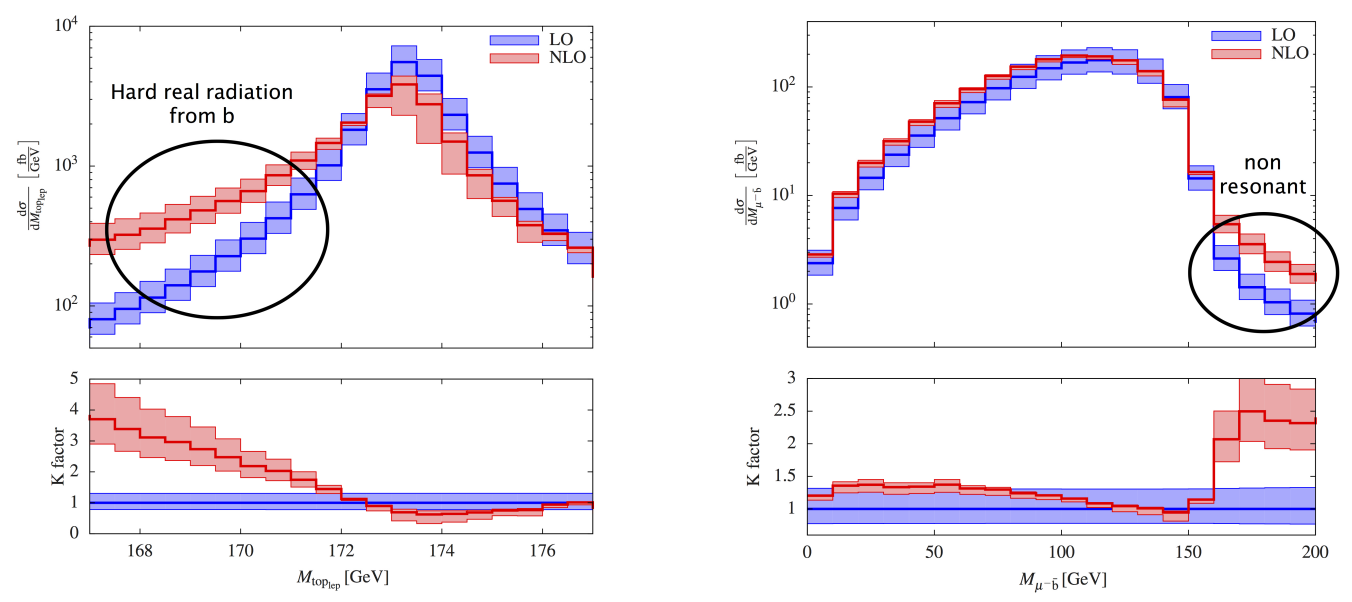

Figure 3: Differential distributions of the reconstructed leptonic top (left) and the decay products (right). Figure taken from [4].

$p p \rightarrow t \bar{t} \rightarrow \mu^{-} \bar{v}_{\mu} b \bar{b} j j$ we discuss the plots of figure 3 , where we can see the invariant mass of the leptonically decaying top quark (left) and the invariant mass of the $\mu^{-} \bar{b}$ system (right). In the $M\left(t_{\text {lep }}\right)$ distribution we see that there is a large $K$-factor below the peak region. Moving from LO to NLO QCD we observe a migration of events to the region below the top mass peak. This is due to hard QCD radiation from the b quark, outside the jet cone radius $\Delta R$. This radiation is not included in the reconstructed b-jet, therefore not included in the top-quark mass reconstruction. This effect is mimicked by the shower even at LO once the latter is included. We further show a key distribution for top-quark mass measurements. In the $M_{\mu^{-} \bar{b}}$ distribution we can see the distinction of the on and off - shell contributions in the steep limit of $M_{\mu^{-} \bar{b}}^{2}=M_{t}^{2}-M_{W}^{2} \approx(154 \mathrm{GeV})^{2}$. The NLO QCD $K$-factor changes before and after this point since for $M_{\mu^{-} \bar{b}} \gtrsim 154 \mathrm{GeV}$ the non doubly resonant diagrams contribute.

\section{Conclusions}

In this proceeding we present the calculations and discuss the results described in [1-4] concerning top-quark pair production and decay process. At the production level we saw that both the NNLOQCD+NLOEW as well as the NNLO+NNLL' calculations soften the spectrum of the $p_{T}$ of the top quark at high $p_{T}$ values and provide a better agreement with the available data. The combination of these two calculations is in progress. We further discussed the merging of the $t \bar{t}+t \bar{t} j$ samples at NLO in QCD+EW under the MEPS@NLO framework. The same effects of the 
EW corrections are observed like in the fixed order calculation and the results are in good agreement with the boosted tops ATLAS analysis. A more detailed comparison between the fixed order NNLOQCD+NLOEW and the MEPS@NLO (QCD+EW) calculations is in progress. Finally we also discussed the semi-leptonic decay of the top-quark pair at NLO QCD accuracy. We pointed out the non trivial differential $K$-factors and the importance of the off - shell contributing Feynman diagrams.

\section{Acknowledgments}

My participation to this workshop was supported by the F.R.S.-FNRS "Fonds de la Recherche Scientifique" (Belgium). I would like also to thank the department of physics at TUM (Munich) for the hospitality during the completion of this project.

\section{References}

[1] M. Czakon, D. Heymes, A. Mitov, D. Pagani, I. Tsinikos and M. Zaro, Top-pair production at the LHC through NNLO QCD and NLO EW, [1705.04105].

[2] M. Czakon, A. Ferroglia, D. Heymes, A. Mitov, B. D. Pecjak, D. J. Scott et al., Resummation for (boosted) top-quark pair production at NNLO+NNLL' in QCD, JHEP 05 (2018) 149, [1803.07623].

[3] C. Gütschow, J. M. Lindert and M. Schönherr, Multi-jet merged top-pair production including electroweak corrections, Eur. Phys. J. C78 (2018) 317, [1803.00950].

[4] A. Denner and M. Pellen, Off-shell production of top-antitop pairs in the lepton+jets channel at NLO QCD, JHEP 02 (2018) 013, [1711.10359].

[5] M. Czakon, M. L. Mangano, A. Mitov and J. Rojo, Constraints on the gluon PDF from top quark pair production at hadron colliders, JHEP 07 (2013) 167, [1303. 7215].

[6] M. Czakon, P. Fiedler and A. Mitov, Total Top-Quark Pair-Production Cross Section at Hadron Colliders Through $O\left(\alpha_{S}^{4}\right)$, Phys. Rev. Lett. 110 (2013) 252004, [1303.6254].

[7] M. Czakon, D. Heymes and A. Mitov, High-precision differential predictions for top-quark pairs at the LHC, Phys. Rev. Lett. 116 (2016) 082003, [1511.00549].

[8] M. Czakon, D. Heymes and A. Mitov, Dynamical scales for multi-TeV top-pair production at the LHC, JHEP 04 (2017) 071, [1606.03350].

[9] S. Moch and P. Uwer, Theoretical status and prospects for top-quark pair production at hadron colliders, Phys. Rev. D78 (2008) 034003, [0 804 . 1476 ].

[10] N. Kidonakis, Two-loop soft anomalous dimensions and NNLL resummation for heavy quark production, Phys. Rev. Lett. 102 (2009) 232003, [0903.2561].

[11] M. Czakon, A. Mitov and G. F. Sterman, Threshold Resummation for Top-Pair Hadroproduction to Next-to-Next-to-Leading Log, Phys. Rev. D80 (2009) 074017, [0 907.1790$].$

[12] V. Ahrens, A. Ferroglia, M. Neubert, B. D. Pecjak and L. L. Yang, Renormalization-Group Improved Predictions for Top-Quark Pair Production at Hadron Colliders, JHEP 09 (2010) 097, [1003.5827]. 
[13] M. Beneke, P. Falgari and C. Schwinn, Threshold resummation for pair production of coloured heavy (s)particles at hadron colliders, Nucl. Phys. B842 (2011) 414-474, [1 007.5414$].$

[14] N. Kidonakis, Next-to-next-to-leading soft-gluon corrections for the top quark cross section and transverse momentum distribution, Phys. Rev. D82 (2010) 114030, [1009.4 935].

[15] V. Ahrens, A. Ferroglia, M. Neubert, B. D. Pecjak and L.-L. Yang, RG-improved single-particle inclusive cross sections and forward-backward asymmetry in $t \bar{t}$ production at hadron colliders, JHEP 09 (2011) 070, [1103.0550].

[16] V. Ahrens, A. Ferroglia, M. Neubert, B. D. Pecjak and L. L. Yang, Precision predictions for the $t+t$ (bar) production cross section at hadron colliders, Phys. Lett. B703 (2011) 135-141, [1105.5824].

[17] M. Beneke, P. Falgari, S. Klein and C. Schwinn, Hadronic top-quark pair production with NNLL threshold resummation, Nucl. Phys. B855 (2012) 695-741, [1109.1536].

[18] M. Cacciari, M. Czakon, M. Mangano, A. Mitov and P. Nason, Top-pair production at hadron colliders with next-to-next-to-leading logarithmic soft-gluon resummation, Phys. Lett. B710 (2012) 612-622, [1111.5869].

[19] B. D. Pecjak, D. J. Scott, X. Wang and L. L. Yang, Resummed differential cross sections for top-quark pairs at the LHC, 1601.07020.

[20] S. Catani, M. Grazzini and H. Sargsyan, Transverse-momentum resummation fo top-quark pair production at the LHC, 1806.01601.

[21] W. Beenakker, A. Denner, W. Hollik, R. Mertig, T. Sack and D. Wackeroth, Electroweak one loop contributions to top pair production in hadron colliders, Nucl. Phys. B411 (1994) 343-380.

[22] J. H. Kühn, A. Scharf and P. Uwer, Electroweak effects in top-quark pair production at hadron colliders, Eur. Phys. J. C51 (2007) 37-53, [hep-ph/0610335].

[23] J. H. Kühn, A. Scharf and P. Uwer, Weak Interactions in Top-Quark Pair Production at Hadron Colliders: An Update, Phys. Rev. D91 (2015) 014020, [1305. 5773].

[24] W. Bernreuther, M. Fuecker and Z. G. Si, Mixed QCD and weak corrections to top quark pair production at hadron colliders, Phys. Lett. B633 (2006) 54-60, [hep-ph/ 0508091$].$

[25] J. M. Campbell, D. Wackeroth and J. Zhou, Study of weak corrections to Drell-Yan, top-quark pair, and dijet production at high energies with MCFM, Phys. Rev. D94 (2016) 093009, [1608. 03356 ].

[26] W. Hollik and D. Pagani, The electroweak contribution to the top quark forward-backward asymmetry at the Tevatron, Phys. Rev. D84 (2011) 093003, [1107.2606].

[27] J. H. Kühn and G. Rodrigo, Charge asymmetries of top quarks at hadron colliders revisited, JHEP 01 (2012) 063, [1109.6830].

[28] A. V. Manohar and M. Trott, Electroweak Sudakov Corrections and the Top Quark Forward-Backward Asymmetry, Phys. Lett. B711 (2012) 313-316, [1201. 3926].

[29] W. Bernreuther and Z.-G. Si, Top quark and leptonic charge asymmetries for the Tevatron and LHC, Phys. Rev. D86 (2012) 034026, [1205.6580].

[30] W. Bernreuther and Z.-G. Si, Distributions and correlations for top quark pair production and decay at the Tevatron and LHC., Nucl. Phys. B837 (2010) 90-121, [1003.3926].

[31] A. Denner and M. Pellen, NLO electroweak corrections to off-shell top-antitop production with leptonic decays at the LHC, JHEP 08 (2016) 155, [1607 . 05571 ]. 
[32] W. Hollik and M. Kollar, NLO QED contributions to top-pair production at hadron collider, Phys. Rev. D77 (2008) 014008, [0 708 .1697].

[33] D. Pagani, I. Tsinikos and M. Zaro, The impact of the photon PDF and electroweak corrections on $t \bar{t}$ distributions, 1606.01915.

[34] I. Tsinikos, EW corrections to t $\bar{t}$ distributions: the photon PDF effect, in 9th International Workshop on Top Quark Physics (TOP 2016) Olomouc, Czech Republic, September 19-23, 2016, 2016. 1611.08234.

[35] I. Tsinikos, EW corrections on top-quark pair production: the impact of the photon PDF, PoS DIS2017 (2018) 138.

[36] T. Jeÿo, J. M. Lindert, P. Nason, C. Oleari and S. Pozzorini, An NLO+PS generator for $t \bar{t}$ and $W t$ production and decay including non-resonant and interference effects, Eur. Phys. J. C76 (2016) 691, [1607.04538].

[37] CMS collaboration, V. Khachatryan et al., Measurement of the differential cross section for top quark pair production in pp collisions at $\sqrt{s}=8$ TeV, Eur. Phys. J. C75 (2015) 542, [1505. 04480 ].

[38] ATLAS collaboration, G. Aad et al., Measurements of top-quark pair differential cross-sections in the lepton+jets channel in pp collisions at $\sqrt{s}=8 \mathrm{TeV}$ using the ATLAS detector, 1511.04716.

[39] J. Alwall, R. Frederix, S. Frixione, V. Hirschi, F. Maltoni, O. Mattelaer et al., The automated computation of tree-level and next-to-leading order differential cross sections, and their matching to parton shower simulations, JHEP 07 (2014) 079, [1405.0301].

[40] R. Frederix, S. Frixione, V. Hirschi, D. Pagani, H. S. Shao and M. Zaro, The automation of next-to-leading order electroweak calculations, 1804.10017.

[41] S. Frixione, V. Hirschi, D. Pagani, H. S. Shao and M. Zaro, Electroweak and QCD corrections to top-pair hadroproduction in association with heavy bosons, JHEP 06 (2015) 184, [15 04.03446 ].

[42] J. R. Andersen et al., Les Houches 2015: Physics at TeV Colliders Standard Model Working Group Report, in 9th Les Houches Workshop on Physics at TeV Colliders (PhysTeV 2015) Les Houches, France, June 1-19, 2015, 2016. 1605.04692.

[43] R. Frederix, S. Frixione, V. Hirschi, D. Pagani, H.-S. Shao and M. Zaro, The complete NLO corrections to dijet hadroproduction, JHEP 04 (2017) 076, [1612. 06548 ].

[44] T. Gehrmann, S. Hoche, F. Krauss, M. Schonherr and F. Siegert, NLO QCD matrix elements + parton showers in $e^{+} e^{-} \longrightarrow$ hadrons, JHEP 01 (2013) 144, [1207.5031].

[45] S. Hoeche, F. Krauss, M. Schonherr and F. Siegert, QCD matrix elements + parton showers: The NLO case, JHEP 04 (2013) 027, [1207.5030].

[46] S. Hoeche, F. Krauss, S. Schumann and F. Siegert, QCD matrix elements and truncated showers, JHEP 05 (2009) 053, [0903. 1219].

[47] ATLAS collaboration, G. Aad et al., Measurement of the differential cross-section of highly boosted top quarks as a function of their transverse momentum in $\sqrt{s}=8 \mathrm{TeV}$ proton-proton collisions using the ATLAS detector, Phys. Rev. D93 (2016) 032009, [1510.03818]. 